\title{
Comparison of the DiagCor GenoFlow Human Papillomavirus Array Test and Roche Linear Array HPV Genotyping Test
}

\author{
Fiona K.Y. Wong ${ }^{\S}$, Johannes C.Y. Ching ${ }^{\S}$ and Joseph K.F. Chow ${ }^{*}$
}

DiagCor Bioscience Inc. Ltd., Hong Kong SAR, China

\begin{abstract}
Persistent infection of high-risk (HR) human papillomavirus (HPV) infection has been widely associated with cervical cancer. Monitoring HPV infection is therefore an important step against cervical cancer development. The DiagCor GenoFlow Human Papillomavirus Array Test (GenoFlow) is a novel HPV test based on PCR and "Flowthrough" hybridization that can identify $33 \mathrm{HPV}$ subtypes in 3 hours. In the present study, the GenoFlow Test was evaluated by comparing the genotyping results of 100 samples with Roche Linear Array HPV Genotyping Test (LA). The tests showed good agreement in detection of HPV-positive samples (concordance rate $=95 \%$, Cohen's Kappa=0.896), with good agreement in detection of HR HPVs (Cohen's Kappa=0.876). The GenoFlow Test showed high sensitivity (95\%), high specificity $(95 \%)$, low false positive rate $(3.33 \%)$ and low false negative rate $(7.50 \%)$. In conclusion, the novel GenoFlow Test showed comparable clinical performance to LA test, and offers advantages of reduction in turnaround time and manpower.
\end{abstract}

Keywords: HPV genotyping test, GenoFlow, Linear Array, Flow-through hybridization.

\section{INTRODUCTION}

Mucosal HPV strains are categorized into high risk (HR) and low risk (LR) strains based on their association with cervical lesions. HR types are more frequently found in premalignant or malignant lesions, while LR types occur more frequently in benign ones such as condylomata acuminate [1]. In a study that included only high quality histological data, infection by HR HPV was demonstrated in $99.7 \%$ of cervical carcinoma [2]. Although the majority of HPV infections can be cleared spontaneously, persistence of HR-HPV infection is a significant risk factor among women in the general screening population for the development of high grade cervical intraepithelial neoplasia and potential progression to cervical cancer [2-7]. Indeed, HR HPV DNA has been detected in $74 \%$ of the premalignant low-grade cervical intraepithelial neoplasia lesions and approximately $84 \%$ of the high grade lesions [8]. On the other hand, persistent infection with the same HPV genotype has also been proved to associate with an elevated risk of developing high-grade preinvasive diseases such as dysplasia and ultimately cervical cancer [7, 9-11].

Despite the prevalent application of Pap smear in some developed countries, cervical cancer remains one of the major female cancers [2], with estimates of 473,000 newly diagnosed cases and 253,500 yearly deaths worldwide [12]. Persistent HR-HPV positivity is related to an enhanced risk of developing premalignant disease and thus these cases should be closely monitored [13, 14]. HPV DNA testing has been shown to be more sensitive than cytology [13, 15-17],

*Address correspondence to this author at the DiagCor Bioscience Inc. Ltd., Hong Kong SAR, China; Tel: (852)-21474088; Fax: (852) 21474138;

E-mail: joseph@diagcor.com

${ }^{\S}$ These authors contributed equally to this study. and is therefore suggested to be useful in monitoring patients with treated cervical intraepithelial neoplasia [13, 18-20]. Several studies have further suggested that HPV DNA testing could improve the efficacy of population-based screening programs in detecting underlying lesions or their subsequent development, either when applied as an independent tool or as an adjunct to Pap smear $[13,14,21$ 23]. Indeed, the incorporation of HPV DNA testing into screening programmes and/or management of women with Atypical Squamous Cells of Undetermined Significance (ASC-US) has been suggested as guidelines in regions including Europe, USA, and Hong Kong [24-26].

Assays targeting HPV viral DNA are commercially available in the market, which vary in running cost, hands on time, specificity and sensitivity. Some of the widely-used assays include the Hybrid Capture 2 (HC2) system (Digene Corporation, Gaithersburg, MD, USA), the AMPLICOR HPV Test (Roche Molecular Diagnostics, Branchburg, NJ, USA), the LINEAR ARRAY HPV Genotyping Test (LA; Roche Molecular Diagnostics, Branchburg, NJ, USA), and the INNO-LiPA HPV Genotyping (Innogenetics, Belgium). Neither HC2 nor the AMPLICOR HPV Test assay is capable of distinguishing specific genotypes, and they also lack the ability to identify infections involving multiple genotypes. Given the facts that 1) oncogenic potential varies among HR-HPVs [27]; 2) risk elevates with persistent infection of the same genotypes [7, 9-11]; and 3) HPV vaccines available could not prevent all types of HPV infections; HPV genotyping is needed in addition to generic screening for the presence or absence of the viruses. The LA and the INNOLiPA HPV Genotyping are both line probe assays capable of identifying 37 and 28 HPV genotypes respectively. However, these assays are rather expensive and they share the intrinsic problem of hybridization, that is, a relatively long turnover time of over 2 hours only from hybridization to colorimetric detection. 
Based on these, we have developed GenoFlow HPV Array test kit (GenoFlow; DiagCor Bioscience, Hong Kong) for HPV genotyping. GenoFlow is a PCR-based assay that co-amplifies the human gene as an internal control to measure the integrity of the sample. This assay, by employing a membrane-based flow-through hybridization technology (US Patent number 5741647), identifies $33 \mathrm{HR}$ and LR HPV viral genotypes $(6,11,16,18,26,31,33,35$, $39,40,42,43,44,45,51,52,53,54,55,56,57,58,59,61$, $66,68,70,71,72,73,81,82,84)$. A novel universal probe was also designed to capture HPV genotypes that were not included in the test panel in order to expand the viral coverage. It has indeed been shown that this universal probe has captured at least 5 other HPV viral subtypes in addition to the 33 genotypes included in the test panel. Through coupling with the flow-through technology platform, a satisfactory sensitivity and specificity has been warranted despite considerable reduction in the reagent cost and handling time [28]. Adding together the high throughput and superiority in handling time as compared to other genotyping assays available in the market, GenoFlow would potentially improve the efficacy of clinical diagnosis. The objective of this study is therefore to validate the performance in sensitivity and specificity of the GenoFlow by comparison with the LA. Our data showed that the GenoFlow attains a comparable performance in both sensitivity and specificity. The other feature of the GenoFlow kit (i.e. reduction in handling time and simultaneously detection of multiple samples) further support its possible role as a good product for HPV genotyping.

\section{MATERIALS AND METHODS}

\section{Study Population and Specimen Collection}

The studied cohort comprised archive samples of 100 women whose cervical scrapes were collected in liquidbased cytology medium (ThinPrep; Cytyc Corp., Marlborough, MA, USA) and sent to DiagCor Bioscience, Hong Kong, for HPV genotyping testing in September to October 2009. In order to increase the proportion of HPV $+v e$ subjects so that the type specificity can be compared between the GenoFlow test and LA test, this study included a panel of HPV positive samples together with random HPV screening samples, making the total number of subjects be 100. The cervical specimens were aliquoted into two aliquots for DNA extraction: one aliquot for GenoFlow test and one aliquot for LA test using the suggested extraction method of each genotyping test.

\section{GenoFlow Human Papillomavirus Array Test Kit}

The GenoFlow test uses biotin-labeled primers and specific probes to detect 33 common HPV types (18 HR HPV types and 15 LR types, Table 1). In this study, DNA was extracted from the aliquot specimen using QIAamp DNA mini kit (Qiagen) according to manufacturer's instructions. The extracted DNA was mixed with PCR reagent mix and DNA Taq Polymerase provided with the GenoFlow test kit and PCR-amplified using the thermocycling condition stated in the manual. The amplicons were genotyped using Flow-through hybridization according to
Table 1. HPV Genotypes Detected by GenoFlow and LA Tests

\begin{tabular}{|c|c|c|c|}
\hline \multirow{2}{*}{ Oncogenic Potential } & \multirow{2}{*}{ HPV Type } & \multicolumn{2}{|c|}{ Detected in ${ }^{\mathrm{a}}$ : } \\
\hline & & GenoFlow & Linear Array \\
\hline \multirow{18}{*}{ High risk } & 16 & $\mathrm{D}$ & $\mathrm{D}$ \\
\hline & 18 & $\mathrm{D}$ & $\mathrm{D}$ \\
\hline & 31 & $\mathrm{D}$ & $\mathrm{D}$ \\
\hline & 33 & $\mathrm{D}$ & $\mathrm{D}$ \\
\hline & 35 & $\mathrm{D}$ & $\mathrm{D}$ \\
\hline & 39 & $\mathrm{D}$ & $\mathrm{D}$ \\
\hline & 45 & $\mathrm{D}$ & $\mathrm{D}$ \\
\hline & 51 & $\mathrm{D}$ & $\mathrm{D}$ \\
\hline & 52 & $\mathrm{D}$ & $\mathrm{D}^{\mathrm{g}}$ \\
\hline & 53 & $\mathrm{D}$ & $\mathrm{D}$ \\
\hline & 56 & $\mathrm{D}$ & $\mathrm{D}$ \\
\hline & 58 & $\mathrm{D}$ & $\mathrm{D}$ \\
\hline & 59 & $\mathrm{D}$ & $\mathrm{D}$ \\
\hline & 66 & $\mathrm{D}^{\mathrm{b}}$ & $\mathrm{D}$ \\
\hline & 68 & $\mathrm{D}^{\mathrm{b}}$ & $\mathrm{D}$ \\
\hline & 73 & $\mathrm{D}$ & $\mathrm{D}$ \\
\hline & 81 & $\mathrm{D}$ & $\mathrm{D}$ \\
\hline & 82 & $\mathrm{D}$ & $\mathrm{D}$ \\
\hline \multirow{22}{*}{ Low risk } & 6 & $\mathrm{D}$ & $\mathrm{D}$ \\
\hline & 11 & $\mathrm{D}$ & $\mathrm{D}$ \\
\hline & 26 & $D^{c}$ & $\mathrm{D}$ \\
\hline & 40 & $\mathrm{D}^{\mathrm{d}}$ & $\mathrm{D}$ \\
\hline & 42 & $\mathrm{D}$ & $\mathrm{D}$ \\
\hline & 43 & $\mathrm{D}$ & --- \\
\hline & 44 & $\mathrm{D}$ & --- \\
\hline & 54 & $\mathrm{D}^{\mathrm{e}}$ & $\mathrm{D}$ \\
\hline & 55 & $\mathrm{D}^{\mathrm{e}}$ & $\mathrm{D}$ \\
\hline & 57 & $D^{f}$ & $\begin{array}{ll}-- \\
--\end{array}$ \\
\hline & 61 & $\mathrm{D}^{\mathrm{d}}$ & $\mathrm{D}$ \\
\hline & 62 & --- & $\mathrm{D}$ \\
\hline & 64 & --- & $\mathrm{D}$ \\
\hline & 67 & --- & $\mathrm{D}$ \\
\hline & 69 & --- & $\mathrm{D}$ \\
\hline & 70 & $\mathrm{D}$ & $\mathrm{D}$ \\
\hline & 71 & $D^{f}$ & $\mathrm{D}$ \\
\hline & 72 & $\mathrm{D}$ & $\mathrm{D}$ \\
\hline & 83 & --- & $\mathrm{D}$ \\
\hline & 84 & $\mathrm{D}^{\mathrm{c}}$ & $\mathrm{D}$ \\
\hline & IS39 & --- & $\mathrm{D}$ \\
\hline & CP6108 & --- & $\mathrm{D}$ \\
\hline
\end{tabular}


manufacturer's instructions. "Flow-through" technology involved hybridization, enzyme conjugation and colorimetric developed and intermittent washings, which can be finished in $35 \mathrm{~min}$. A sample will be considered valid only if the hybridization control (for monitoring the success of hybridization), amplification control (for access of sample integrity or successful PCR reaction), and/or specific HPV type on the membrane were obtained. Positivity was shown by colored dots on the membrane, which was recorded by scanning of the membrane by flat based scanner.

\section{Linear Array HPV Genotyping Test}

The LA test uses biotinylated primers to amplify the polymorphic L1 region of HPV genome $(\sim 450 \mathrm{bp})$ and human $\beta$-globin (for DNA integrity). It identifies $18 \mathrm{HR}$ HPV types and 19 LR types (Table 1). The LA test in this study was carried out by a third party in order to rule out possible bias in this study. DNA was extracted from specimen aliquot by AmpliLute liquid medium extraction kit (Roche Molecular Diagnostics, Branchburg, NJ, USA). The PCR amplicons were denatured by denaturation solution and subjected to hybridization with a single LA HPV genotyping strip that was coated with HPV type-specific and human $\beta$ globin probes according to manufacturer's instructions. The biotin-labeled amplicons hybridized to the probes only if the type-specific sequence matched the amplicons. The biotinlabeled amplicons were detected by colorimetric development and the results were read visually by comparing the pattern of colored lines to the provided reference guide.

\section{Statistical Analysis}

To compare the GenoFlow test and LA test, the studied cases were categorized into two groups: the HPV +ve and HPV -ve groups by each test. The agreement of the tests in the detection of HPV + ve cases was assessed by Cohen's Kappa statistic, with values higher than 0.8 indicate excellent agreement. The Kappa value was calculated using an online program (http://faculty.vassar.edu/lowry/kappa.html). The sensitivity, specificity, false positive (+ve) rate, false negative (-ve) rate, and concordant rate of GenoFlow test were calculated according to the formulae in Table 3, using the results of LA genotyping test as reference. To further study the agreement of the test in detecting HR +ve cases, the cases were further categorized into three groups: the HR group, LR group and HPV -ve group by each genotyping test. A case was categorized into HR group if it showed any of the HR types; a case with multiple infections of both HR and LR types was also categorized into HR group. Cohen's Kappa statistic was used to assess the agreement of both genotyping tests.

\section{RESULTS}

The GenoFlow test and the LA test detect 33 and 37 HPV genotypes, respectively. Table $\mathbf{1}$ showed the genotypes detected by both tests. The concordance between the GenoFlow and LA tests in the detection of HPV infection is shown in Table 2. Among the 100 women tested, $57 \%$ were found HPV-positive by both tests and 38\% were found HPVnegative by both tests, with an overall agreement of $95 \%$ (Cohen's Kappa $=0.896$ [95\% CI: 0.808-0.985]). Using the results of LA test as reference, the sensitivity (95.00\%), specificity $(95.00 \%)$, false positive rate $(3.33 \%)$, false negative rate $(7.50 \%)$, and overall concordance rate $(95 \%)$ of GenoFlow test were calculated as shown in Table 3. The high Kappa value (0.896) and the high sensitivity and specificity illustrated the good agreement between GenoFlow test and LA test.

Table 2. Concordance Between DiagCor GenoFlow Test and Roche LA Test in 100 Samples

\begin{tabular}{|c|c|c|c|}
\hline \multirow{2}{*}{ LA Test } & \multicolumn{2}{|c|}{ No. of Samples GenoFlow Test } & \multirow{2}{*}{ Total } \\
\hline & Positive & Negative & \\
\hline Positive & 57 & 3 & 60 \\
\hline Negative & 2 & 38 & 40 \\
\hline Total & 59 & 41 & 100 \\
\hline
\end{tabular}

Table 3. The Sensitivity and Specificity of DiagCor GenoFlow Test

\begin{tabular}{|c|c|c|}
\hline & Formula & \\
\hline \hline Sensitivity & $\mathrm{TP} /(\mathrm{TP}+\mathrm{FN})$ & $95.00 \%$ \\
\hline Specificity & $\mathrm{TN} /(\mathrm{TN}+\mathrm{FP})$ & $95.00 \%$ \\
\hline False positive rate & $\mathrm{FP} /(\mathrm{TP}+\mathrm{FN})$ & $3.33 \%$ \\
\hline False negative rate & $\mathrm{FN} /(\mathrm{TN}+\mathrm{FP})$ & $7.50 \%$ \\
\hline Concordance rate & $(\mathrm{TP}+\mathrm{TN}) /(\mathrm{TP}+\mathrm{TN}+\mathrm{FP}+\mathrm{FN})$ & $95.00 \%$ \\
\hline Note: $\mathrm{TP}:$ true positive; $\mathrm{TN}:$ true negative; $\mathrm{FP}$ : true positive; $\mathrm{FN}$ : false negative.
\end{tabular}

Concerning the concordance between the kits in the overall infection profile, 29 samples were completely concordant cases that showed complete match of genotypes by both tests among the 57 samples found positive by both tests. The remaining 28 samples were compatible cases that involved multiple infections. The profiles resulted by both tests were shown in Table 4.

In order to assess the capability of the GenoFlow test in distinguishing HR and LR cases, the positive results of both tests are categorized into HR and LR groups, with HR group contained the samples showing any HR genotype. The concordance of the GenoFlow and LA tests is shown in Table 5, with a Cohen's Kappa value 0.876 (95\% CI: 0.787 $0.965)$. This indicated a good agreement between the tests, with a very slight decrease in Kappa compared to the value in Table 2 (Cohen's kappa $=0.896,95 \%$ CI: 0.808-0.985), showing that the capability of diagnosing high-risk HPV infection is similar for both tests.

\section{DISCUSSION}

In this study, we have shown that the GenoFlow test and the LA test are highly congruent for the genotypes detectable in both assays, albeit with minor dissimilarities in compatible cases. The overall concordance of the two assays was $95 \%$, with a Cohen's Kappa value of 0.896 , which indicated a good correlation. Among the 100 samples, 67 were concordant cases that showed complete agreement, in 
Table 4. Compatible Cases in this Study

\begin{tabular}{|c|c|c|}
\hline \multirow{2}{*}{ Identifier } & \multicolumn{2}{|c|}{ HPV Genotypes } \\
\hline & GenoFlow Test & LA Test \\
\hline 2 & 52 & $52 *, 66$ \\
\hline 3 & $40 / 61$ & 40,51 \\
\hline 5 & 16 & 16,70 \\
\hline 8 & $6,26 / 84,51,59,82$ & $6,18,39,51,59,82,84$ \\
\hline 13 & 35 & $35,52 *, 62$ \\
\hline 14 & 82 & 51,82 \\
\hline 22 & 52,81 & $40,52 *, 56,66,81$ \\
\hline 23 & $43 / 44,57 / 71$ & $40,62,71$ \\
\hline 26 & 35 & $35,52 *$ \\
\hline 27 & 82 & 51,82 \\
\hline 28 & $52,66 / 68$ & $26,52 *, 66$ \\
\hline 29 & $43 / 44,57 / 71,81$ & $55,71,81$ \\
\hline 31 & $6,40 / 61,59$ & 59 \\
\hline 35 & 58 & $16,52 *, 58$ \\
\hline 36 & 52 & $52^{*}, 62$ \\
\hline 38 & 18 & $18,39,62$ \\
\hline 41 & 70 & 54,70 \\
\hline 48 & $11,39,53,81$ & $39,53,81$ \\
\hline 49 & 39 & 39,83 \\
\hline 58 & 58 & $52^{*}, 58$ \\
\hline 59 & $33,53,58,59$ & $35,52 *, 53,58$ \\
\hline 60 & 51 & 40,51 \\
\hline 65 & 16,52 & $16,52 *, 62$ \\
\hline 66 & 58 & $52 *, 58$ \\
\hline 68 & 51,53 & $39,51,53,82$ \\
\hline 70 & 33 & $18,33,52 *, 58$ \\
\hline 90 & $16,33,53,66 / 68$ & $16,33,52 *, 66$ \\
\hline 93 & 53 & 39,53 \\
\hline
\end{tabular}

Table 5. Analysis of High-Risk and Low-Risk Cases

\begin{tabular}{|c|c|c|c|c|}
\hline & & \multicolumn{3}{|c|}{ GenoFlow Test } \\
\hline & & High-Risk & Low-Risk & Negative \\
\hline \multirow{3}{*}{$\stackrel{\vec{s}}{\stackrel{5}{\Xi}}$} & High-risk & 49 & 1 & 1 \\
\hline & Low-risk & 1 & 6 & 2 \\
\hline & Negative & 2 & 0 & 38 \\
\hline
\end{tabular}

Note: Cohen's Kappa $=0.876$ (95\% CI: 0.787-0.965).

Results were further analyzed by categorizing into high risk and low risk cases. Grey boxes indicate cases with concordance between the two assays.

which 29 positive cases were identically concluded by the two assays and the other 38 cases were HPV negative; 5 samples were discordantly concluded. The GenoFlow test was able to identify HPV 16 and 18, which together caused about $70 \%$ cervical cancer $[29,30]$; as well as HPV 58, which was prevalent in east Asia and especially in China [31-33].

Cases that involved unique genotypes detected by either of the assays but not by both were defined as "compatible cases". All of the 28 compatible cases involved multiple infections. GenoFlow test did not detect 17 genotypes in 26 cases (sample number 2, 3, 5, 8, 13, 14, 22, 23, 26-29, 35, $36,38,41,49,58-60,65,66,68,70,90$ and 93); but it detected 6 genotypes that were not detected by LA in 6 cases (sample number 23, 29, 31, 48, 59 and 90) (Table 4). Nevertheless, among the 26 cases that consisted of genotypes not detected by GenoFlow test, sample number 26, 58 and 66 actually brought about ambiguity concerning the presence the HPV 52. The HPV $52 *$ probe of LA test detected HPV 52, 33, 35 and 58, and the infection of HPV 52 cannot be negated nor guaranteed with the positivity of either of the 33-, 35-, or 58- specific probes. Therefore, the possibility of these cases of having complete agreement between both tests could not be ruled out. These results (complete agreement between HPV profiles- 67\%; compatible- 28\%; discordant: $5 \%$ ) were comparable to an earlier study comparing LA and another commercially available assay [34] (complete agreement between HPV profiles- $65.9 \%$; compatible- $21.2 \%$; discordant: $12.9 \%$ ). Variations among genotyping results have been reported in many commercial kits or assays as reported in earlier studies $[34,35]$. It has even been reported that the complete concordance of HPV infection profiles generated by the same LA assay was only $83 \%$ [36]. The complexity of assessing multiple genotypes has been addressed in earlier studies that biased amplification would possibly occur if certain genotypes are present in molar excess [35]. Nevertheless, it is important to identify whether such variation would lead to faulty diagnostic conclusions of HPV status, that is, whether patients with HR HPV infection would be classified as LR HPV infection, or vice versa. This study therefore further analyzed the results by separating the cases into HR and LR (Table 5). Among the 100 cases, 49, 6, and 38 cases were concordantly identified as HR, LR and negative respectively. Two cases were concluded by the assays with different risk levels (2\%); 1 LA HR (1\%) and 2 LR $(2 \%)$ case were concluded negative by GenoFlow test; and 2 GenoFlow test HR cases (2\%) were concluded negative by LA test. This analysis resulted in a Cohen's Kappa value of 0.876 , which indicated a good correlation between the tests in delineating HR and LR cases.

The reliability of GenoFlow test was evidenced by its good correlation with LA test as indicated in this study. In consideration of the assay protocols, both the LA and GenoFlow test consist of four main processes: DNA extraction, PCR amplification of targeted sequences, hybridization of PCR products to specific oligonucleotide probes on a membrane, and colorimetric detection [34, 37]. Indeed, despite the reliability conferred, the relatively long turnaround time and manual operation are the common drawback of any assays that employ hybridization. For example, it has been estimated by a previous study that the hybridization process (i.e. excluding PCR) in manual LA test requires $180 \mathrm{~min}$, of which incubation takes at least $107 \mathrm{~min}$ 
[38]. Flow-through system was developed to enhance the efficiency yet retain the reliability of hybridization technique in a simple operation setting. It has been estimated that with the application of the Flow-through system, the PCR and hybridization of HPV identification can be completed within 3 hours [28].

The recommended methods of DNA extraction of both tests make use of column extraction. The PCR of both tests requires less than 2.5 hours to complete when set according to respective manual of the kits. Nevertheless, the hybridization process of the tests requires significantly different duration because of the use of Flow-through technology. The incubation time in the hybridization process of GenoFlow test requires about $35 \mathrm{~min}$, compared to LA test, which requires about $107 \mathrm{~min}$ according to its manual (Table 6). In addition, GenoFlow test facilitates aspiration of solution from 15 reactions at the same time by Flow-through technology while conventional methods require aspiration one by one reaction. The kits are also different in the number of solutions requiring fresh preparation, which would affect the ease of handling and hands-on time. All solutions provided with GenoFlow test are ready-to-use while 6 solutions in LA test require fresh preparation before use from stock solutions provided.

Table 6. Incubation Times of GenoFlow Test and LA Test

\begin{tabular}{|c|c|c|c|}
\hline \multicolumn{2}{|c|}{ GenoFlow Test } & \multicolumn{2}{c|}{ LA Test } \\
\hline Step & $\begin{array}{c}\text { Time } \\
(\mathbf{m i n})\end{array}$ & Step & $\begin{array}{c}\text { Time } \\
(\mathbf{m i n})\end{array}$ \\
\hline \hline Pre-hybridization & 2 & Hybridization & 30 \\
\hline Incubation & 5 & Fast ambient wash & $\sim 1$ \\
\hline Hybridization wash $\mathrm{x} 3$ & $\sim 1.5$ & Stringent wash & 15 \\
\hline Temperature change and Blocking & $\sim 10$ & Conjugate & 30 \\
\hline Enzyme conjugation & 3.5 & Fast ambient wash & $\sim 1$ \\
\hline Temperature change and wash $\mathrm{x} 4$ & $\sim 5$ & Ambient wash $\mathrm{x} 2$ & 20 \\
\hline Detection & 5 & Citrate & 5 \\
\hline Wash $\mathrm{x} 3$ & $\sim 1.5$ & Substrate & 5 \\
\hline Stop & 1 & H $2 \mathrm{O}$ & 0 \\
\hline Total & $\sim 35$ & Total & $\sim 107$ \\
\hline
\end{tabular}

The need of screening a large number of samples is observed in current market, especially due to the promotion in prevention of cervical cancer worldwide, such as the population-based screening programmes launched in at least 13 European countries [39]. The capability of Flow-through system in processing 15 samples simultaneously further enhances screening efficacy by processing more samples at a short turnaround time and reduced manpower.

In conclusion, this study indicated that the GenoFlow test is an applicable and accurate assay for HPV genotyping in clinical samples. The performance of GenoFlow test is also comparable to LA test. Adding together the simplicity and reduced turnaround time associated to its protocol, GenoFlow test holds significant potential to serve as an affordable alternative in HPV diagnosis and screening programme.

\section{ACKNOWLEDGEMENTS}

The authors would also like to thank Dr. Joseph WO Tam and Mr. Richard Y.M. Ma for their constructive advice to this article.

\section{CONFLICT OF INTEREST STATEMENT}

The authors declare competing interests. They are employees of DiagCor Bioscience Incorporation Limited.

\section{REFERENCES}

[1] Munoz N, Bosch FX, de Sanjose S, et al. Epidemiologic classification of human papillomavirus types associated with cervical cancer. N Engl J Med 2003; 348: 518-27.

[2] Walboomers JM, Jacobs MV, Manos MM, et al. Human papillomavirus is a necessary cause of invasive cervical cancer worldwide. J Pathol 1999; 189: 12-9.

[3] Schiffman MH, Bauer HM, Hoover RN, et al. Epidemiologic evidence showing that human papillomavirus infection causes most cervical intraepithelial neoplasia. J Natl Cancer Inst 1993; 85: 95864.

[4] Bosch FX, Lorincz A, Munoz N, Meijer CJ, Shah KV. The causal relation between human papillomavirus and cervical cancer. J Clin Pathol 2002; 55: 244-65.

[5] Bosch FX, Manos MM, Munoz N, et al. Prevalence of human papillomavirus in cervical cancer: a worldwide perspective. International biological study on cervical cancer (IBSCC) Study Group. J Natl Cancer Inst 1995; 87: 796-802.

[6] Munoz N, Bosch FX. The causal link between HPV and cervical cancer and its implications for prevention of cervical cancer. Bull Pan Am Health Organ 1996; 30: 362-77.

[7] Wallin KL, Wiklund F, Angstrom T, et al. Type-specific persistence of human papillomavirus DNA before the development of invasive cervical cancer. N Engl J Med 1999; 341: 1633-8.

[8] Melchers WJ, Bakkers JM, Wang J, et al. Short fragment polymerase chain reaction reverse hybridization line probe assay to detect and genotype a broad spectrum of human papillomavirus types. Clinical evaluation and follow-up. Am J Pathol 1999; 155: $1473-8$.

[9] Ho GY, Burk RD, Klein S, et al. Persistent genital human papillomavirus infection as a risk factor for persistent cervical dysplasia. J Natl Cancer Inst 1995; 87: 1365-71.

[10] Kjaer SK, van den Brule AJ, Paull G, et al. Type specific persistence of high risk human papillomavirus (HPV) as indicator of high grade cervical squamous intraepithelial lesions in young women: population based prospective follow up study. BMJ 2002; 325: 572 .

[11] Remmink AJ, Walboomers JM, Helmerhorst TJ, et al. The presence of persistent high-risk HPV genotypes in dysplastic cervical lesions is associated with progressive disease: natural history up to 36 months. Int J Cancer 1995; 61: 306-11.

[12] National Cervical Cancer Coalition. 2008. [cited 2009 November 2]; Available from: http://www.nccc-online.org/.

[13] Carozzi FM, Confortini M, Cecchini S, et al. Triage with human papillomavirus testing of women with cytologic abnormalities prompting referral for colposcopy assessment. Cancer 2005; 105: $2-7$.

[14] Lorincz AT, Richart RM. Human papillomavirus DNA testing as an adjunct to cytology in cervical screening programs. Arch Pathol Lab Med 2003; 127: 959-68.

[15] Bollmann R, Bankfalvi A, Griefingholt $\mathrm{H}$, et al. Validity of combined cytology and human papillomavirus (HPV) genotyping with adjuvant DNA-cytometry in routine cervical screening: results from 31031 women from the Bonn-region in West Germany. Oncol Rep 2005; 13: 915-22.

[16] Dalla Palma P, Pojer A, Girlando S. HPV triage of women with atypical squamous cells of undetermined significance: a 3-year experience in an Italian organized programme. Cytopathology 2005; 16: 22-6. 
[17] Mayrand MH, Duarte-Franco E, Rodrigues I, et al. Human papillomavirus DNA vs Papanicolaou screening tests for cervical cancer. N Engl J Med 2007; 357: 1579-88.

[18] Bais AG, Rebolj M, Snijders PJ, et al. Triage using HPV-testing in persistent borderline and mildly dyskaryotic smears: proposal for new guidelines. Int J Cancer 2005; 116: 122-9.

[19] Chao A, Lin CT, Hsueh S, et al. Usefulness of human papillomavirus testing in the follow-up of patients with high-grade cervical intraepithelial neoplasia after conization. Am J Obstet Gynecol 2004; 190: 1046-51.

[20] Sarian LO, Derchain SF, Andrade LA, Tambascia J, Morais SS, Syrjanen KJ. HPV DNA test and Pap smear in detection of residual and recurrent disease following loop electrosurgical excision procedure of high-grade cervical intraepithelial neoplasia. Gynecol Oncol 2004; 94: 181-6.

[21] The Atypical Low-Grade Squamous Intraepithelial Lesions Triage Study Group. Human papillomavirus testing for triage of women with cytologic evidence of low-grade squamous intraepithelial lesions: baseline data from a randomized trial. The Atypical Squamous Cells of Undetermined Significance/Low-Grade Squamous Intraepithelial Lesions Triage Study (ALTS) Group. J Natl Cancer Inst 2000; 92: 397-402.

[22] Cuzick J, Szarewski A, Terry G, et al. Human papillomavirus testing in primary cervical screening. Lancet 1995; 345: 1533-6.

[23] Denise Zielinski G, Snijders PJ, Rozendaal L, et al. High-risk HPV testing in women with borderline and mild dyskaryosis: long-term follow-up data and clinical relevance. J Pathol 2001; 195: 300-6.

[24] HKCOG. Guidelines on the management of an abnormal cervical smear. Hong Kong; 2002 [cited 2009 December 19]; Available from:

http://hkcog.obg.cuhk.edu.hk/docs/collegeVंguidelines/Managemen t\%20of\%20Abnormal\%20Cervical\%20Smear\%20RevisedV̈2002.p df.

[25] Jordan J, Martin-Hirsch P, Arbyn M, et al. European guidelines for clinical management of abnormal cervical cytology, part 2 . Cytopathology 2009; 20: 5-16.

[26] Saslow D, Runowicz CD, Solomon D, et al. American Cancer Society guideline for the early detection of cervical neoplasia and cancer. CA Cancer J Clin 2002; 52: 342-62.

[27] Castle PE, Solomon D, Schiffman M, Wheeler CM. Human papillomavirus type 16 infections and 2-year absolute risk of cervical precancer in women with equivocal or mild cytologic abnormalities. J Natl Cancer Inst 2005; 97: 1066-71.
[28] Chow KF. A membrane-based flow-through hybridization technology: a rapid and versatile tool for molecular diagnostics. Open Biotech J 2008; 2: 22-8.

[29] Munoz N, Bosch FX, Castellsague X, et al. Against which human papillomavirus types shall we vaccinate and screen? The international perspective. Int J Cancer 2004; 111: 278-85.

[30] Schiffman M, Castle PE, Jeronimo J, Rodriguez AC, Wacholder S. Human papillomavirus and cervical cancer. Lancet 2007; 370: 890907.

[31] Chan PK, Li WH, Chan MY, Ma WL, Cheung JL, Cheng AF. High prevalence of human papillomavirus type 58 in Chinese women with cervical cancer and precancerous lesions. J Med Virol 1999; 59: 232-8.

[32] Chen CA, Liu CY, Chou HH, et al. The distribution and differential risks of human papillomavirus genotypes in cervical preinvasive lesions: A Taiwan Cooperative Oncologic Group Study. Int J Gynecol Cancer 2006; 16: 1801-8.

[33] Huang S, Afonina I, Miller BA, Beckmann AM. Human papillomavirus types 52 and 58 are prevalent in cervical cancers from Chinese women. Int J Cancer 1997; 70: 408-11.

[34] van Hamont D, van Ham MA, Bakkers JM, Massuger LF, Melchers WJ. Evaluation of the SPF10-INNO LiPA human papillomavirus (HPV) genotyping test and the roche linear array HPV genotyping test. J Clin Microbiol 2006; 44: 3122-9.

[35] van Doorn LJ, Quint W, Kleter B, et al. Genotyping of human papillomavirus in liquid cytology cervical specimens by the PGMY line blot assay and the $\operatorname{SPF}(10)$ line probe assay. J Clin Microbiol 2002; 40: 979-83.

[36] Steinau M, Swan DC, Unger ER. Type-specific reproducibility of the Roche linear array HPV genotyping test. J Clin Virol 2008; 42: 412-4.

[37] Stevens MP, Rudland E, Garland SM, Tabrizi SN. Assessment of MagNA pure LC extraction system for detection of human papillomavirus (HPV) DNA in PreservCyt samples by the Roche AMPLICOR and LINEAR ARRAY HPV tests. J Clin Microbiol 2006; 44: 2428-33.

[38] Stevens MP, Garland SM, Tabrizi SN. Validation of an automated detection platform for use with the roche linear array human papillomavirus genotyping test. J Clin Microbiol 2008; 46: 3813-6.

[39] Anttila A, von Karsa L, Aasmaa A, et al. Cervical cancer screening policies and coverage in Europe. Eur J Cancer 2009; 45: 2649-58.

This is an open access article licensed under the terms of the Creative Commons Attribution Non-Commercial License (http://creativecommons.org/licenses/by-nc/ 3.0/) which permits unrestricted, non-commercial use, distribution and reproduction in any medium, provided the work is properly cited. 